\title{
Rickettsia rickettsii infection causes apoptotic death of cultured cerebellar granule neurons
}

Rickettsia rickettsii is a small, Gram-negative, obligate intracellular bacillus and the causative agent of a fulminating human disease, Rocky Mountain spotted fever (RMSF) (Silverman, 1984). RMSF is a tick-borne disease wherein rickettsiae grow in the gut lining of ticks, often without harming the host. Human infection results either from a tick bite (commonest) or rarely by contamination of cut skin or a wound with faeces of the ticks (Bleck, 1999). In humans, $R$. rickettsii grows principally in endothelial cells of small blood vessels, producing vasculitis, thrombosis of vessels, fever, typical skin rash (petechial), organ dysfunctions and cell death (Walker \& Raoult, 2000). Although the primary target of $R$. rickettsii is the vascular endothelial cell, often there is multi-organ and system involvement in the later stages of RMSF pathogenesis.

The central nervous system (CNS) appears to be one of the major systems involved during the later stages of RMSF pathogenesis (Walker et al., 2001). During infection, the incidence of major dysfunctions pertaining to the CNS has been summarized as headache (79-91\%), signs of meningoencephalitis and meningismus (18\%), stupor (21-26\%), ataxia (5-18\%), seizures (8\%), hearing loss (7\%), coma (9-10\%) and death (4-8\%) (Walker \& Raoult, 2000). In RMSF, restlessness and irritability often lead to mental confusion and delirium, followed by coma in later stages of the disease. Cortical blindness, central deafness, spastic paralysis and sixth nerve palsy have also been reported (Walker et al., 2001). Among all rickettsial diseases, RMSF has emerged as an important cause of status epilepticus in children in the USA (Bleck, 1999). A confirmed case of acute disseminated encephalomyelitis after RMSF has also been reported (Wei \& Baumann, 1999). For RMSF, among the non-human hosts and reservoirs required to complete the natural cycle are rodents, dogs and rabbits (Bleck, 1999).
The cerebellum is involved in important functions, mainly dealing with balance, voluntary muscle coordination, and gait, so damage to the cerebellum may lead to loss of these functions. Ataxia, hypotonia, paresis and paralysis are frequently found to be associated with cerebellar dysfunction. As similar types of symptoms are observed in the later stages of RMSF (Bleck, 1999), one may suspect the involvement of the cerebellum. In order to investigate whether cerebellar neurons are infected or damaged during $R$. rickettsii infection, we used primary cultures of rat cerebellar granular neurons (CGNs). Our results demonstrate that $R$. rickettsii efficiently infects neuronal cells and that the infection causes apoptotic death of neurons.

Cultures of human umbilical vein endothelial cells (ECs) were established as described previously (Joshi et al., 2003; Silverman, 1984). Near-confluent cultures of ECs at second passage were used for various experiments. Primary cultures of cerebellar granule cells were prepared from 8-day-old Sprague-Dawley rat cerebellum essentially as described previously (Kovács

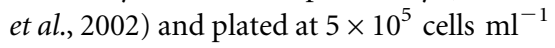
into 24-well plates ( $1 \mathrm{ml}$ per well). Cytosine- $\beta$-arabinofuranoside (Ara-C; $10 \mu \mathrm{M}$ ) was added $24 \mathrm{~h}$ after plating to limit the number of non-neuronal cells.

A plaque-purified seed stock $\left(1 \times 10^{7}-5 \times 10^{7}\right.$ p.f.u. $\left.\mathrm{ml}^{-1}\right)$ of $R$. rickettsii (Sheila Smith strain) was prepared from infected Vero cells as described previously (Joshi et al., 2003; Silverman, 1984). The endothelial cells or CGNs were infected with $\sim 5 \times 10^{4}$ p.f.u. $R$. rickettsii diluted in $80 \mu$ culture medium per $\mathrm{cm}^{2}$ of cell culture area. After $2 \mathrm{~h}$ incubation, the medium containing infective organisms was aspirated, and the cell monolayer was washed twice to remove Vero cell debris and then retained quickly in fresh culture medium for the remainder of infection. CGN cultures were quickly retained in conditioned (pre-saved) medium. Infection of ECs plated on Thermanox coverslips (Nalge-Nunc International) was monitored in parallel by staining with polyclonal anti-rickettsia serum [anti-RR; Centers for Disease Control and Prevention (CDC)] by indirect immunofluorescence, as described elsewhere (Clifton et al., 1998; Silverman, 1984). Some of the cultures were exposed to $30 \mu \mathrm{g}$ tetracycline $/ \mathrm{ml}^{-1}$ for $30 \mathrm{~min}$ prior to, and then maintained in throughout, the infection to inactivate $R$. rickettsii (Silverman, 1984). In some experiments, incubation of uninfected cells with staurosporine (STS; $2 \mu \mathrm{M}$ ) was included as a positive control for induction of apoptotic cell death (Joshi et al., 2003).

For immunocytochemical analyses, ECs or CGNs plated on glass coverslips (as described above) were left uninfected or infected with $R$. rickettsii in the presence or absence of tetracycline, or treated with apoptosis-inducing agent(s). The cells were then processed for fixation, permeabilization, blocking and washing, essentially as described previously (Joshi et al., 2003), for specific identification of a neuronal marker ( $\beta$-III tubulin) and/or $R$. rickettsii-infected cells, with the following primary antibodies: mouse anti- $\beta$-III tubulin (anti-TUJ1) (1:300; Covance) or anti- $R$. rickettsii rabbit polyclonal serum ( $1: 1000$; CDC). In the case of double labelling, first the cell-bearing coverslips were treated with anti-TUJ1, followed by treatment with the corresponding secondary antibodies $\left(1: 400,37^{\circ} \mathrm{C}, 1 \mathrm{~h}\right)$. The coverslips were then stained with anti- $R$. rickettsii rabbit polyclonal serum ( $1: 1000$, $37^{\circ} \mathrm{C}, 1 \mathrm{~h}$ ) followed by the corresponding secondary antibodies $\left(1: 200,37^{\circ} \mathrm{C}\right.$, $30 \mathrm{~min})$. Secondary antibodies were either rhodamine-conjugated or fluorescein-conjugated goat anti-rabbit or anti-mouse IgG (Calbiochem) or Texas-red-conjugated anti-mouse IgG (Southern Biotechnology Associates). The coverslips were then processed for examination by fluorescent, bright-field or phase-contrast microscopy. 
The extent of apoptotic cell death of immunocytochemically identified neurons or ECs was determined by terminal deoxynucleotidyl transferase-mediated digoxigenin-dUTP nick-end labelling (TUNEL) assay. The use of TUNEL has been reported to detect nuclear DNA fragmentation during the course of apoptotic cell death. TUNEL was performed using the ApopTag-Fluorescein in situ apoptosis detection kit (Intergen) as per the manufacturer's directions and as described previously (Joshi et al., 2003). A minimum of 500 cells, located in multiple, randomly selected fields, were counted for each condition in three different sets of experiments, and data were calculated as the arithmetic mean values of percentages of TUNEL-positive cells.

Fluorescent, phase-contrast or bright-field microscopy was performed, and digitalized images were captured using an Olympus CR40 fluorescent microscope and a Qimaging cooled digital charge-coupled device (CCD) video camera, with appropriate settings. Digitalized images were saved as TIFF files and edited using Adobe Photoshop (version 6.0). The datasets in this study were calculated as a percentage of the corresponding control levels. Statistical significance was determined by one-way ANOVA followed by Bonferroni's test for multiple comparisons.

To test whether $R$. rickettsii infects CGNs from a non-human rodent host, rat primary CGN cultures on day 8 in vitro were exposed to $R$. rickettsii. A double

immunofluorescent-staining technique was employed to identify infected neurons, using anti-RR serum and a neuron-specific (anti-neuronal $\beta$-III isoform of tubulin; anti-TUJ1) antibody. Fig. 1 shows that CGNs were infected ( $\sim 65 \%$ of the neurons) during a $6 \mathrm{~h}$ exposure period to $R$. rickettsii, and the infection induced widespread neuronal degeneration, as evident from the bright-field images. Overlay of the anti-RR and neuron-specific anti-TUJ1 staining of the same field
(Fig. 1f) demonstrates that $R$. rickettsii infects neurons. The small arrows in the bright-field image of the same field (Fig. 1c) point to infected neurons, immunopositive for both $R$. rickettsii and neuron-specific $\beta$-III tubulin. Some of the neuronal cells are heavily infected and degenerated (large arrowheads), but weakly immunopositive for the neuron-specific $\beta$-III tubulin, most likely because the advanced degeneration and/or bacterial overload has destroyed or masked the epitope recognized by the monoclonal anti-TUJ1 antibody. To verify that the observed neuronal degeneration was $R$. rickettsii-induced, CGN cultures were exposed to $R$. rickettsii in the presence of tetracycline, an antibacterial agent, which efficiently kills $R$. rickettsii and has been in use for this purpose. As expected, the neurons exposed to $R$. rickettsii in the presence of tetracycline remained intact (data not shown).

R. rickettsii infection has been shown to cause host endothelial-cell apoptosis by interfering with cellular apoptotic

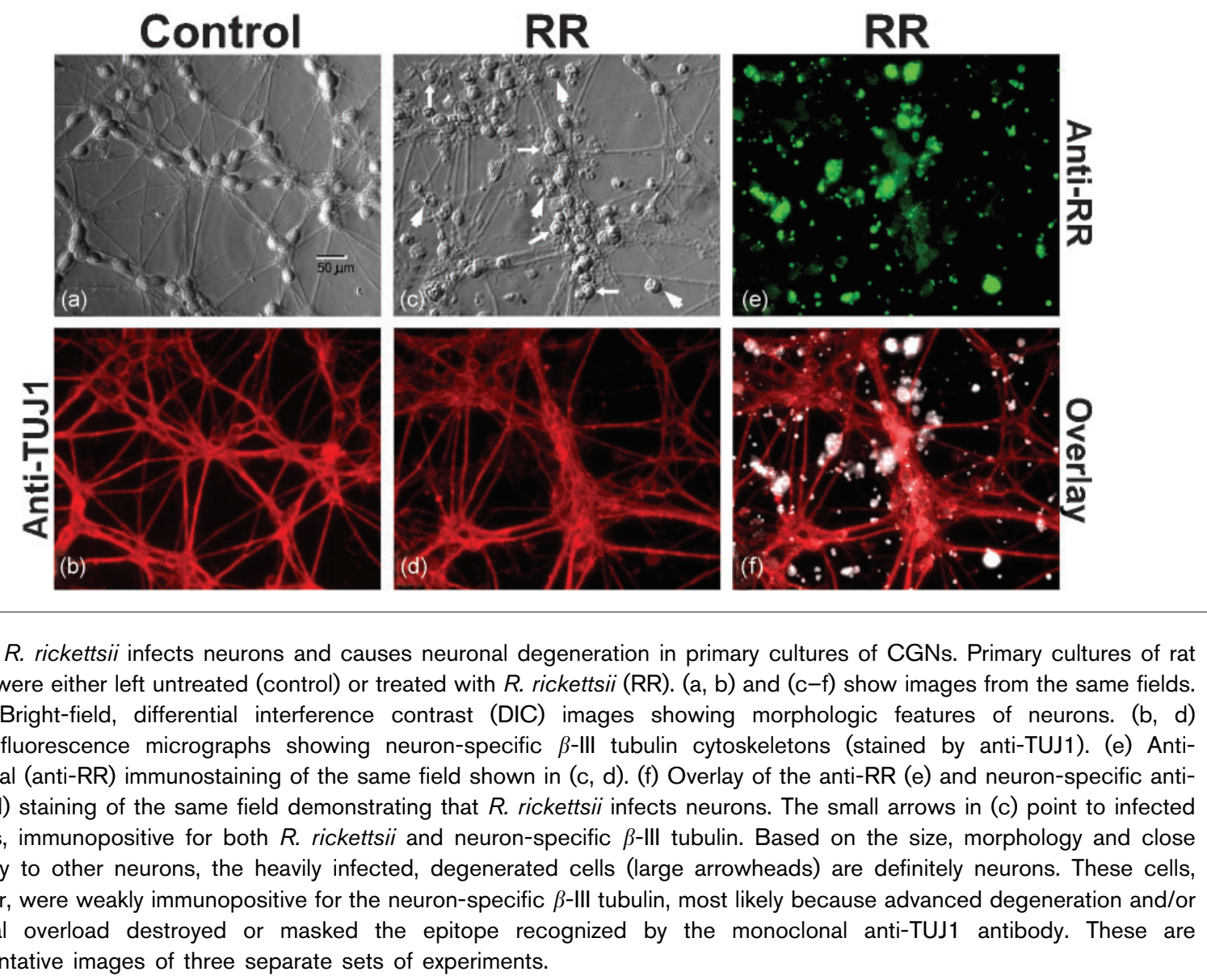

Fig. 1. R. rickettsii infects neurons and causes neuronal degeneration in primary cultures of CGNs. Primary cultures of rat CGNs were either left untreated (control) or treated with $R$. rickettsii (RR). ( $a, b)$ and (c-f) show images from the same fields. $(a, c)$ Bright-field, differential interference contrast (DIC) images showing morphologic features of neurons. (b, d) Immunofluorescence micrographs showing neuron-specific $\beta$-III tubulin cytoskeletons (stained by anti-TUJ1). (e) Antirickettsial (anti-RR) immunostaining of the same field shown in (c, d). (f) Overlay of the anti-RR (e) and neuron-specific antiTUJ1 (d) staining of the same field demonstrating that $R$. rickettsii infects neurons. The small arrows in (c) point to infected neurons, immunopositive for both $R$. rickettsii and neuron-specific $\beta$-III tubulin. Based on the size, morphology and close proximity to other neurons, the heavily infected, degenerated cells (large arrowheads) are definitely neurons. These cells, however, were weakly immunopositive for the neuron-specific $\beta$-III tubulin, most likely because advanced degeneration and/or bacterial overload destroyed or masked the epitope recognized by the monoclonal anti-TUJ1 antibody. These are representative images of three separate sets of experiments. 
machinery (Clifton et al., 1998; Joshi et al., $2003,2004)$. Therefore, to investigate whether neurons also undergo apoptotic death upon infection, we performed TUNEL assays. Endothelial cells, which are the primary target cells of the pathogen during pathogenesis of RMSF, served for comparison. Fig. 2(a) shows that $R$. rickettsii exposure induced apoptotic cell death in both endothelial cells and CGNs, and that the number of TUNEL-positive cells was markedly reduced by tetracycline co-treatment. STS-treated cultures were also included as a positive control for cell-death induction. We verified nuclear DNA changes peculiar to apoptosis in the cells by Hoechst 33342 and propidium iodide (PI) staining, and the results demonstrated the absence of necrotic changes, but the presence of apoptotic changes, very similar to the results obtained by the TUNEL assay (data not shown).

Fig. 2(b) demonstrates the results of quantitative analyses by the TUNEL assay. $R$. rickettsii infection induced significant apoptosis of immunocytochemically identified neurons $(P<0.001)$ and of endothelial cells $(P<0.001)$ compared with uninfected (control) cells. About $52 \%$ of the infected neurons were TUNEL positive. CGNs were more vulnerable to infectioninduced cell death than endothelial cells ( $P$ $<0.001)$. Tetracycline co-treatment significantly reduced the extent of apoptotic death of neurons $(P=0.001)$ and endothelial cells $(P<0.01)$.

It is evident from the clinical signs and symptoms that the CNS is involved during the later stages of the pathogenesis of RMSF (Bleck, 1999). The CNS is also a crucial target in many other rickettsial diseases (Bleck, 1999). The classical cases of cerebellitis have been reported for Mediterranean spotted fever caused by Rickettsia conorii (Alioua et al., 2003), and involvement of key enzymes leading to cellular injury upon in vivo infection has been demonstrated earlier (Rydkina et al., 2004). It has been demonstrated by a specific immunohistochemistry technique, during the development of mouse-model studies, that rickettsiae reach brain tissues when injected intravenously (Rydkina et al., 2004). This therefore demonstrates that rickettsiae cross the blood-brain barrier to reach brain tissues. To the best of our knowledge, there is no report of experimental in vitro $R$. rickettsii infection of neuronal cells. In the present study, we tested whether cultured neurons are sensitive to $R$. rickettsii infection. Our results show that neurons are efficiently infected, and that the infection causes significant neuronal apoptotic cell death. We included R. rickettsii infection of ECs as a control for infection and infection-induced apoptotic cell death (Clifton et al., 1998). CGNs were significantly more vulnerable to $R$. rickettsii infection-induced apoptotic cell death than endothelial cells during these experiments (see Fig. 2b). Cellular or tissue vulnerability depends on the type of cell and the type of pathogen (Knodlar \& Finlay, 2001); therefore, differences in $R$. rickettsii infection-induced apoptosis in different cell types signify pathological susceptibility. $R$. rickettsii has shown differential susceptibilities to types of tissues and organs (Rydkina et al., 2004).

Of numerous techniques available to study and quantify apoptosis in cell culture, only a few are useful when applied to pathogen-invaded isolated cells. Among these, the TUNEL assay remains the most (a)
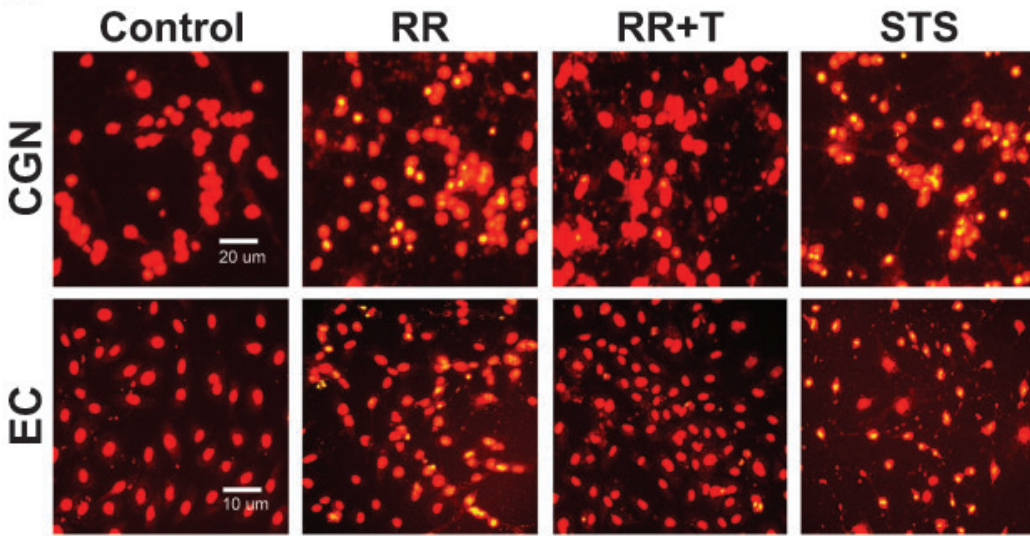

(b)

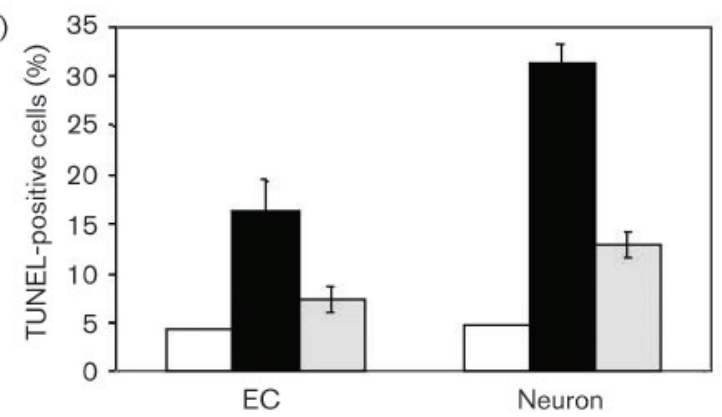

Fig. 2. (a) $R$. rickettsii infection induces cell death, as detected by the TUNEL assay. EC or CGN cultures were either left untreated (control) or treated with $R$. rickettsii in the absence (RR) or presence (RR+T) of tetracycline. A high incidence of nuclear DNA fragmentation was seen in rickettsia-treated cells, which was significantly reduced by tetracycline co-treatment. STS-treated cultures were included as a positive control for apoptosis induction. (b) Analysis and score of TUNELpositive cells; a graphical representation of the scores of TUNEL-positive endothelial cells in EC cultures and immunocytochemically identified neurons in CGN cultures. $R$. rickettsii infection (black bars) induced a significant amount of nuclear DNA fragmentation in neurons $(P=0.001)$ and endothelial cells $(P$ $<0.001)$ compared with uninfected cultures (white bars). Tetracycline co-treatment (grey bars) significantly reduced the number of TUNEL-positive neurons $(P=0.001)$ and endothelial cells $(P<0.01)$. Bars and error bars represent mean \pm SEM $(n=3)$. Statistical significance was determined by one-way ANOVA followed by Bonferroni's test for multiple comparisons. 
widely used technique (Kelly et al., 2003). It takes advantage of the multiple free DNA ends generated by activated endonucleases to insert labelled dUTP that can later be detected by light or fluorescence microscopy; the ease and power of the technique has led to its wide acceptance. In necrosis, there is uptake of PI (indicating damage to the cell membrane), and lack of nuclear condensation and fragmentation, but in apoptosis, a lack of PI uptake (indicating the integrity of the cell membrane) and the presence of nuclear condensation and/or fragmentation. Although simultaneous staining with Hoechst 33342 and PI excludes the possibility of necrotic death, TUNEL assays are much superior to the former technique, although it stains apoptotic cells with a high degree of specificity and sensitivity (Kelly et al., 2003). Therefore, we preferred TUNEL assays, which detect early to moderate apoptotic nuclear changes very easily.

R. rickettsii is highly sensitive to tetracycline and its derivatives (Breitschwerdt et al., 1999). It is one of the drugs of choice for effective therapy against RMSF diseases (CDC, 2000). Tetracycline has also been shown to inactivate $R$. rickettsii, resulting in a drastic decrease in the in vitro infection of cultured cells (Silverman, 1984). Therefore, we used tetracycline treatments in some of the experimental conditions to inhibit $R$. rickettsii infection and also to confirm that the viability of $R$. rickettsii is required for in vitro infection of the cells under study (Silverman, 1984). Viable rickettsiae have been reported to be required for host-cell infection and underlying cellular events during pathogenesis (Rydkina et al., 2004). Tetracycline effectively reduced both infection (data not shown) and infection-induced apoptotic death in EC and CGN cultures (see Fig. 2b), confirming that the significant cellular degeneration detected was due to $R$. rickettsii infection. Further studies are being planned to elucidate the early cellular responses and underlying apoptotic signalling cascade.

To the best of our knowledge, this is the first report demonstrating that $R$. rickettsii efficiently infects cultured neurons and that the infection is sufficient to cause apoptotic cell death.

\section{Acknowledgements}

We thank Dr S. K. Sahni and Dr S. B. Maggirwar for their wholehearted technical support. We acknowledge the help given by Li Hua and Servio H. Ramirez during this piece of work.

\section{Suresh G. Joshi ${ }^{1} \dagger$ and Attila D. Kovács ${ }^{2}$ \\ ${ }^{1}$ Hematology and Oncology Unit, Department of Medicine, University of Rochester Medical Center, School of Medicine and Dentistry, Rochester, NY 14642, USA \\ ${ }^{2}$ Department of Microbiology and Immunology, University of Rochester Medical Center, School of Medicine and Dentistry, Rochester, NY 14642, USA}

†Present address: Room JAH-314, Division of Infectious Diseases, Department of Medicine, Thomas Jefferson University, 1020 Locust St, Philadelphia, PA 19107, USA.

Correspondence: Suresh G. Joshi (surejoshi@yahoo.com; suresh.joshi@jefferson.edu)

Alioua, Z., Bourazza, A., Lamsyah, H., Erragragui, Y., Boudi, O., Karouach, K., Ghfir, M., Mossedaq, R. \& Sedrati, O. (2003).

Neurological features of Mediterranean spotted fever: a study of four cases. Rev Med Interne 24, 824-829 (in French).

Bleck, T. P. (1999). Central nervous system involvement in rickettsial diseases. Cent Nerv Syst Infect 17, 801-811.

Breitschwerdt, E. B., Papich, M. G., Hegarty, B. C., Gilger, B., Hancock, S. I. \& Davidson, M. G. (1999). Efficacy of doxycycline, azithromycin, or trovafloxacin for treatment of experimental Rocky Mountain spotted fever in dogs. Antimicrob Agents Chemother 43, 813-821.

CDC (2000). Consequences of delayed diagnosis of Rocky Mountain spotted fever in children - West Virginia, Michigan, Tennessee, and Oklahoma, May-July 2000. JAMA ( $\mathrm{Am}$ Med Assoc) 284, 2049-2050.

Clifton, D. R., Goss, R. A., Sahni, S. K., Van Antwerp, D., Baggs, R. B., Marder, V. J., Silverman, D. J. \& Sporn, L. A. (1998). NF- $\kappa$ B-dependent inhibition of apoptosis is essential for host cell survival during Rickettsia rickettsii infection. Proc Natl Acad Sci U S A 95, 4646-4651.

Joshi, S. G., Francis, C. W., Silverman, D. J. \& Sahni, S. K. (2003). Nuclear factor-kappa B protects against host cell apoptosis during Rickettsia rickettsii infection by inhibiting activation of apical and effector caspases and maintaining mitochondrial integrity. Infect Immun 71, 4127-4136.

Joshi, S. G., Francis, C. W., Silverman, D. J. \& Sahni, S. K. (2004). NF- $\kappa$ B activation suppresses host cell apoptosis during Rickettsia rickettsii infection via regulatory effects on intracellular localization or levels of apoptogenic and antiapoptotic proteins. FEMS Microbiol Lett 234, 333-341.

Kelly, K. J., Sandoval, R. M., Dunn, K. W., Molitoris, B. A. \& Dagher, P. C. (2003). A novel method to determine specificity and sensitivity of the TUNEL reaction in the quantitation of apoptosis. Am J Physiol Cell Physiol 284, C1309-C1318.

Knodlar, L. A. \& Finlay, B. B. (2001). Salmonella and apoptosis: to live or let die? Microbes Infect 3, 1321-1326.

Kovács, A. D., Cebers, G., Cebere, A. \& Liljequist, S. (2002). Selective and AMPA receptor-dependent astrocyte death following prolonged blockade of glutamate reuptake in rat cerebellar cultures. Exp Neurol 174, 58-71.

Rydkina, E., Sahni, S. K., Santucci, L. A., Turpin, L. C., Baggs, R. B. \& Silverman, D. J. (2004). Selective modulation of antioxidant enzyme activities in host tissues during Rickettsia conorii infection. Microb Pathog 36, 293-301.

Silverman, D. J. (1984). Rickettsia rickettsiiinduced cellular injury of human vascular endothelium in vitro. Infect Immun 44, 545-553.

Walker, D. H. \& Raoult, D. (2000). Rickettsia rickettsii and other spotted fever group rickettsiae (Rocky Mountain spotted fever and other spotted fevers). In Principles and Practice of Infectious Diseases, vol. 2, pp. 2035-2042. Edited by G. L. Mandell, J. E. Bennett \& R. Dolin. Philadelphia, PA: Churchill Livingstone.

Walker, D., Raoult, D., Dumler, J. S. \& Marrie, T. (2001). Rickettsial diseases. In Harrison's Principles of Internal Medicine, vol. 1, pp. 1065-1073. Edited by E. Braunwald, A. S. Fauci, D. L. Kasper, S. L. Hauser, D. L. Longo \& J. L. Jameson. New York: McGraw-Hill.

Wei, T. Y. \& Baumann, R. J. (1999). Acute disseminated encephalomyelitis after Rocky Mountain spotted fever. Pediatr Neurol 21, 503-505. 